\title{
Investigation of nanoscale morphological changes in organic photovoltaics during solvent vapor annealing
}

\author{
Steve Miller, ${ }^{* a}$ Giovanni Fanchini, ${ }^{* a}$ Yun-Yue Lin, ${ }^{b}$ Cheng Li, ${ }^{c}$ Chun-Wei Chen, ${ }^{b}$ Wei-Fang Su ${ }^{b}$ and \\ Manish Chhowalla ${ }^{a}$
}

Received 11th September 2007, Accepted 1st November 2007

First published as an Advance Article on the web 20th November 2007

DOI: $10.1039 / \mathrm{b} 713926 \mathrm{~h}$

Improvement of the photovoltaic efficiency via exposure of organic poly(3-hexylthiophene) $(\mathrm{P} 3 \mathrm{HT})$ and phenyl- $\mathrm{C}_{61}$-butyric acid methyl ester (PCBM) devices to solvent vapor at room temperature is reported. In situ photoluminescence (PL) and Raman spectroscopy, in conjunction with ex situ optical absorption and atomic force microscopy, have been used to provide insight into the nanoscale morphological changes occurring during solvent vapor annealing. We found that in $1: 1$ composites of P3HT : PCBM, suppression of PL, narrowing in line-width of the $1442 \mathrm{~cm}^{-1}$ P3HT Raman peak, and strong modifications in the optical absorption spectra were observed during solvent vapor annealing, while minimal changes occurred in pure P3HT films. We attribute these spectral modifications to de-mixing of PCBM and subsequent stacking of $\mathrm{P} 3 \mathrm{HT}$ in coplanar conjugated segments, similar to what is observed during thermal annealing.

\section{Introduction}

Organic photovoltaics are promising alternatives to their inorganic counterparts ${ }^{1}$ because they can be prepared simply from solution on flexible substrates which, in principle, should compensate for their lower efficiency. The most successful organic photovoltaic system to date exploits the properties of the soluble fullerene derivative, phenyl- $\mathrm{C}_{61}$-butyric acid methyl ester (PCBM), as an electron acceptor and a conducting polymer, poly(3-hexylthiophene) (P3HT), as an electron donor. ${ }^{1-14}$ Efficiencies of solar cells based on bulk heterojunctions of P3HT and PCBM have been reported to range from 3-5\% 7,9 when devices are prepared on rigid substrates in inert environments. There is great experimental interest in P3HT : PCBM and other organic photovoltaic devices because theoretical studies have predicted efficiencies as high as $11 \%{ }^{15}$

Thermal annealing of P3HT : PCBM bulk heterojunction devices is a critical step for improving their efficiency. ${ }^{8,9}$ It has been demonstrated (through post experimental analysis) that thermal annealing strongly affects the morphology of the nanocomposite layer via de-mixing of $\mathrm{PCBM}^{16,17}$ and stacking of P3HT in coplanar conjugated segments. ${ }^{4,16}$ In thermal annealing, relatively high temperatures ranging from $120{ }^{\circ} \mathrm{C}$ to $150{ }^{\circ} \mathrm{C}$ are often used, ${ }^{1,2,9}$ which limits the use of some inexpensive plastic substrates. Recently, Dickey et al. ${ }^{18}$ reported an improvement in the performance of organic 3-ethyl-silylethynyl anthradithiophene (TES ADT) transistors via room temperature solvent vapor annealing (that is, exposing the deposited organic layers to solvent vapor).

\footnotetext{
${ }^{a}$ Rutgers University, Materials Science and Engineering, 607 Taylor Road, Piscataway, NJ 08854, USA. E-mail: stevemil@eden.rutgers.edu; fanchini@rci.rutgers.edu

${ }^{b}$ National Taiwan University, Department of Materials Science and Engineering, Taipei, Taiwan, Republic of China

${ }^{c}$ New Jersey Institute of Technology, Department of Chemistry and

Environmental Science, Newark, NJ 07102, USA
}

Electroluminescence of P3HT has also been shown to improve with exposure to $\mathrm{CHCl}_{3}$ vapor. ${ }^{19}$ The beneficial effect of better structural order attained through solvent vapor annealing suggests that the same method could also improve the performance of P3HT : PCBM solar cells. Indeed, recently it has been reported that exposing $\mathrm{P} 3 \mathrm{HT}$ : $\mathrm{PCBM}$ photovoltaic devices to solvent vapor along with a subsequent thermal annealing step leads to an improvement in efficiency. ${ }^{20}$ The improvement is better than thermal annealing alone. It has also been demonstrated that slow evaporation of the solvent leads to a dramatic improvement in performance. ${ }^{7}$ The latter may be comparable to solvent vapor annealing since the slow evaporation soaks the organic layer in the solvent vapor, allowing the P3HT : PCBM to rearrange in an ordered manner. Although slow evaporation of solvent, referred to as solvent annealing, was first reported by Li et al., ${ }^{7}$ we demonstrate that exposure to solvent vapor even after the films have dried leads to an improvement in device performance. An additional difference between our work and that reported in ref. 7 is that annealing with the solvent vapor leads to improvement in device properties within less than a minute in contrast to slow evaporation which can take up to 20 minutes. Finally, the most significant difference between our work and previous reports is that we clearly demonstrate spectroscopic effects occurring during solvent vapor annealing and through in situ Raman spectroscopy show that ordering of the P3HT matrix is likely to be the primary cause of the enhancement in device performance. Although the use of solvent vapor annealing to improve device performance has been previously reported, little is known about the morphological transformations occurring during such a process, and on the spectroscopic signatures which might be used to recognize them.

In this paper, we elucidate the mechanisms responsible for the improvement in P3HT : PCBM photovoltaic cell efficiency by solvent vapor annealing. We have utilized in situ Raman and photoluminescence spectroscopies to monitor structural 
and optoelectronic changes in the P3HT : PCBM during the annealing. In addition, we have employed ex situ ultravioletvisible (UV-VIS) spectroscopy and atomic force microscopy (AFM) to characterize the P3HT : PCBM nano-composites. Specifically, the structural changes occurring during solvent vapor annealing of P3HT : PCBM solar cells in solvent vapor at room temperature are described. The efficiencies of the devices tested in this study increased from $0.8 \%$ (for as fabricated devices) to $2.6 \%$ (with some devices achieving efficiencies up to $3.0 \%$ ) after solvent vapor annealing, comparable to values we routinely obtain after thermal annealing. Furthermore, both the morphological changes and the maximum efficiency of P3HT : PCBM devices were found to saturate within 30 seconds to 3 minutes of exposure to the solvent vapor.

\section{Experimental}

The organic solar cells were prepared by depositing a mixture of as-received P3HT (regioregularity 98.5\%, Rieke Metals, Inc.) and PCBM (purity $>99.5 \%$, American Dye Source, Inc.) on to an indium-tin oxide (ITO) layer $\left(<20 \Omega \square^{-1}\right.$ resistance, $>85 \%$ transmittance) on poly(ethylene terephthalate) and glass substrates covered with a $50 \mathrm{~nm}$ thick poly(3,4-ethylenedioxythiophene) : poly(styrenesulfonate) (PEDOT : PSS) layer. Specifically, a $10 \mathrm{mg} \mathrm{mL}^{-1}$ solution at $1: 1$ weight ratio of P3HT to PCBM in chloroform or chlorobenzene was spincoated at $1000 \mathrm{rpm}$, resulting in a $100-120 \mathrm{~nm}$ active layer. Thermally evaporated aluminium was used for the top contact electrodes. The photovoltaic characteristics were evaluated under a Newport AM1.5 solar simulator operated at $100 \mathrm{~mW} \mathrm{~cm}{ }^{-2}$. External quantum efficiency (EQE) measurements were performed using a halogen light source with an Oriel Cornerstone 130 monochrometer and Newport 1830-C power meter.

The in situ morphological and optoelectronic changes in the P3HT : PCBM layers were observed by monitoring the changes in the Raman and photoluminescence (PL) features using a Renishaw InVia spectrometer operating at $1.57 \mathrm{eV}$ excitation (power: $1 \mathrm{~mW}$ ). Each spectrum was recorded using a 3 second integration time and care was taken to avoid laser soaking of the sample. The experimental configuration used to perform solvent vapor annealing while simultaneously measuring the PL and Raman features is shown in Fig. 1a. A special sample holder was fabricated in which the substrate was placed upside down in the top cap of a Petri dish filled with the solvent. This allowed the laser to pass through the PET and ITO layers from the top to the P3HT : PCBM layer while being exposed to the solvent vapor. The choice of a low excitation energy $\left(E_{\mathrm{i}}=1.57 \mathrm{eV}\right)$ strongly favors the observation of the Raman and PL arising from P3HT while suppressing the PL from PCBM which has a much larger optical gap. While $1.57 \mathrm{eV}$ is below the optical absorption edge of P3HT, ${ }^{21}$ a broad PL background was identified in both P3HT and P3HT : PCBM samples. Control measurements on PET, ITO and PCBM alone were performed in order to discount the contribution to the PL from these materials in the system. No similar background could be identified in any material other than P3HT. Additionally, all measurements (a)
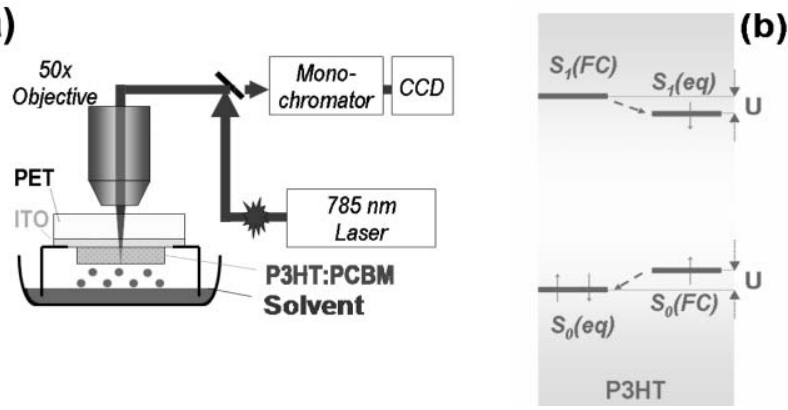

Fig. 1 (a) Schematic of the experimental configuration used to perform solvent vapor annealing while simultaneously measuring the PL and Raman features. (b) Schematic of radiative recombination mechanism of polaron-exciton pairs into Franck-Condon (FC) states (adapted from ref. 27).

were repeated after annealed samples had been thoroughly dried in air to ensure that there was no contribution to the PL from the solvent. Although the exact origin of the PL in P3HT using $1.57 \mathrm{eV}$ excitation is debatable, the intensity of the P3HT : PCBM PL background varied sub-linearly with laser power in the $0.01-1 \mathrm{~mW}$ range, suggesting the material is weakly absorbing due to mid-gap defect mediated states and subsequent radiative decay. ${ }^{22-24}$

PL in conjugated thiophenes has been suggested ${ }^{25-27}$ to arise from radiative recombination of polaron-exciton pairs into Franck-Condon (FC) states, as sketched in Fig. 1b. Therefore, the shift of the PL peak with respect to the excitation energy provides information about the polaron-exciton binding energy ( $2 U$, as indicated in Fig. $1 \mathrm{~b}) .{ }^{27}$ Therefore, PL spectroscopy can be a powerful tool for the investigation of the nature, the binding energy, and the degree of confinement of the electronic states in photoexcited P3HT. It should be noted that for some of our in situ Raman and PL measurements reported here, the PEDOT : PSS layer was omitted in order to avoid masking of P3HT features which overlap with those of PEDOT. Additional films of pure P3HT were also deposited and analyzed for comparison. Tapping mode atomic force microscopy (AFM) (Digital Instruments Nanoscope) and optoelectronic measurements (using a Perkin-Elmer Lambda 20 transmittance spectrophotometer for the solutions and a Jobin-Yvon UVISEL spectral ellipsometer coupled with a multilayer model for the solid films) were also performed.

\section{Results and discussion}

\subsection{Photovoltaic efficiencies}

The current density versus voltage curves under AM1.5 illumination $\left(100 \mathrm{~mW} \mathrm{~cm}^{-2}\right)$ for a photovoltaic device fabricated and solvent vapor annealed using chlorobenzene are shown in Fig. 2a. Similar characteristics and improvements in efficiency were also obtained for devices fabricated with and annealed in chloroform. Typical diode characteristics are observed for before and after solvent annealing measurements. A close observation of the high current region at positive voltages indicates that the vapor annealing leads to an improvement in transport due to ordering of the P3HT (see discussion below). The short circuit current increases by more 

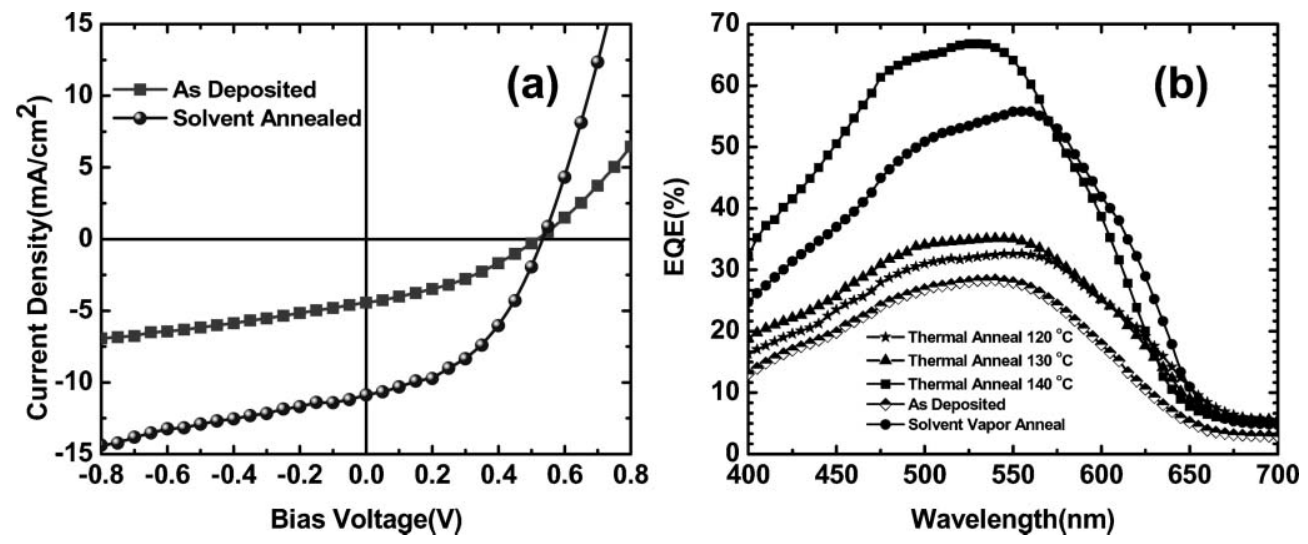

Fig. 2 (a) Current density versus voltage plot for P3HT : PCBM photovoltaic devices before (squares) and after 2 minutes of solvent vapor annealing (circles) with chlorobenzene. (b) External quantum efficiency (EQE) measurements for as deposited, solvent vapor annealed and thermally annealed devices.

than twofold (from $\sim 4.4$ to $10.9 \mathrm{~mA} \mathrm{~cm}^{-2}$ ) while the fill factor increased from $36 \%$ to $44 \%$, resulting in an efficiency of approximately $2.6 \%$, comparable to other reports in the literature. ${ }^{20}$ Efficiency of thermally annealed (performed under standard conditions up to $140{ }^{\circ} \mathrm{C}$ as described in ref. 28) devices was approximately $2.8 \%$. The EQE measurements for as deposited, solvent annealed and thermally annealed devices are plotted in Fig. 2b. The solvent vapor annealed device shows a maximum EQE of $\sim 56 \%$ (at $555 \mathrm{~nm}$ ), twice that of the as deposited device which had an EQE of $\sim 28 \%$ (at $540 \mathrm{~nm}$ ). Similar improvements in EQE can be seen across the entire spectrum below approximately $650 \mathrm{~nm}$ which suggests improved exciton dissociation and charge transport attributable to increased order in the matrix. While the maximum EQE for a thermally annealed device was $\sim 67 \%$, this device required an annealing temperature of $140{ }^{\circ} \mathrm{C}$ which may not suitable for most flexible substrates without incurring damage or degrading the material life expectancy. EQE measurements for devices thermally annealed at temperatures less than $140{ }^{\circ} \mathrm{C}$ were lower than those for solvent annealed devices, as shown in Fig. 2b. The highest efficiency values were obtained on ITO/glass substrates for both annealing treatments. It must also be mentioned that of the numerous devices we tested, both types of annealing treatments (thermal and solvent vapor) yielded similar improvements in efficiency. The maximum value of efficiency we obtained was $3 \%$ but most devices yielded values of $\sim 2.6 \%$. We have found that efficiencies of devices prepared under similar conditions can vary from $2.0 \%$ to $3.0 \%$. The wide variation in device performance is typical for our devices and likely related to factors such as aging of raw materials and exposure to air during measurements.

\subsection{Optoelectronic spectroscopy}

Further information on the mechanism responsible for the enhancement in efficiency by solvent vapor annealing can be inferred from additional ex situ optoelectronic characterization of the devices. Qualitatively, we observed that the color of P3HT films spin-coated in the absence of PCBM is purple and does not change upon solvent vapor annealing. In contrast, the
P3HT : PCBM films are orange immediately after spin coating but become purple during the first 30 seconds of solvent vapor annealing. These effects can be quantitatively verified using UV-VIS absorption spectroscopy. The optical absorption spectra of as deposited thin films and their respective starting solutions are compared in Fig. 3a. It can be observed that the absorption edges of the P3HT : PCBM solution and thin films are similar. In contrast, the optical absorption edge of the P3HT films is strongly red-shifted. The effect of solvent vapor annealing on the absorption spectra of the thin films is compared in Fig. 3b. It can be seen that the spectra of pure P3HT, which are red-shifted during solidification (Fig. 3a), are insensitive to solvent vapor annealing. In contrast, for the P3HT : PCBM films the absorption coefficient above $\sim 510 \mathrm{~nm}$ is observed to red-shift upon solvent vapor annealing and is comparable to that of pure P3HT. It can also be observed from Fig. $3 b$ that the absorption features related to PCBM do not change upon solvent vapor annealing and are present only below $400 \mathrm{~nm}$ (i.e. $>3 \mathrm{eV}){ }^{29}$ Thus, the changes in the observed optical absorption edges during solidification and solvent vapor annealing are mainly due to structural modifications in the P3HT, in agreement with observations of Chirvase et al. ${ }^{6}$ and others. ${ }^{30}$ Further support for the structural changes described above can also be seen in the development of the shoulder near $600 \mathrm{~nm}$ in P3HT : PCBM films which is absent in the as deposited P3HT : PCBM film. However, upon solvent vapor annealing, this shoulder becomes apparent in the P3HT : PCBM film with an absorption coefficient of $\sim 2 \times$ $10^{4} \mathrm{~cm}^{-1}$. Higher absorption at this wavelength has been described as a vibronic feature generally explained by increased ordering of intra-chain interactions and higher crystallization in P3HT. ${ }^{31,32}$ Such structural ordering, although prevented during the deposition of P3HT : PCBM films, is favored during solvent vapor annealing and provides beneficial effects on the photovoltaic device efficiencies.

\subsection{Atomic force microscopy}

The physical changes in the morphology of the P3HT : PCBM films before and after solvent vapor annealing observed by AFM are shown in Fig. $3 c$ and $d$, respectively. The as 

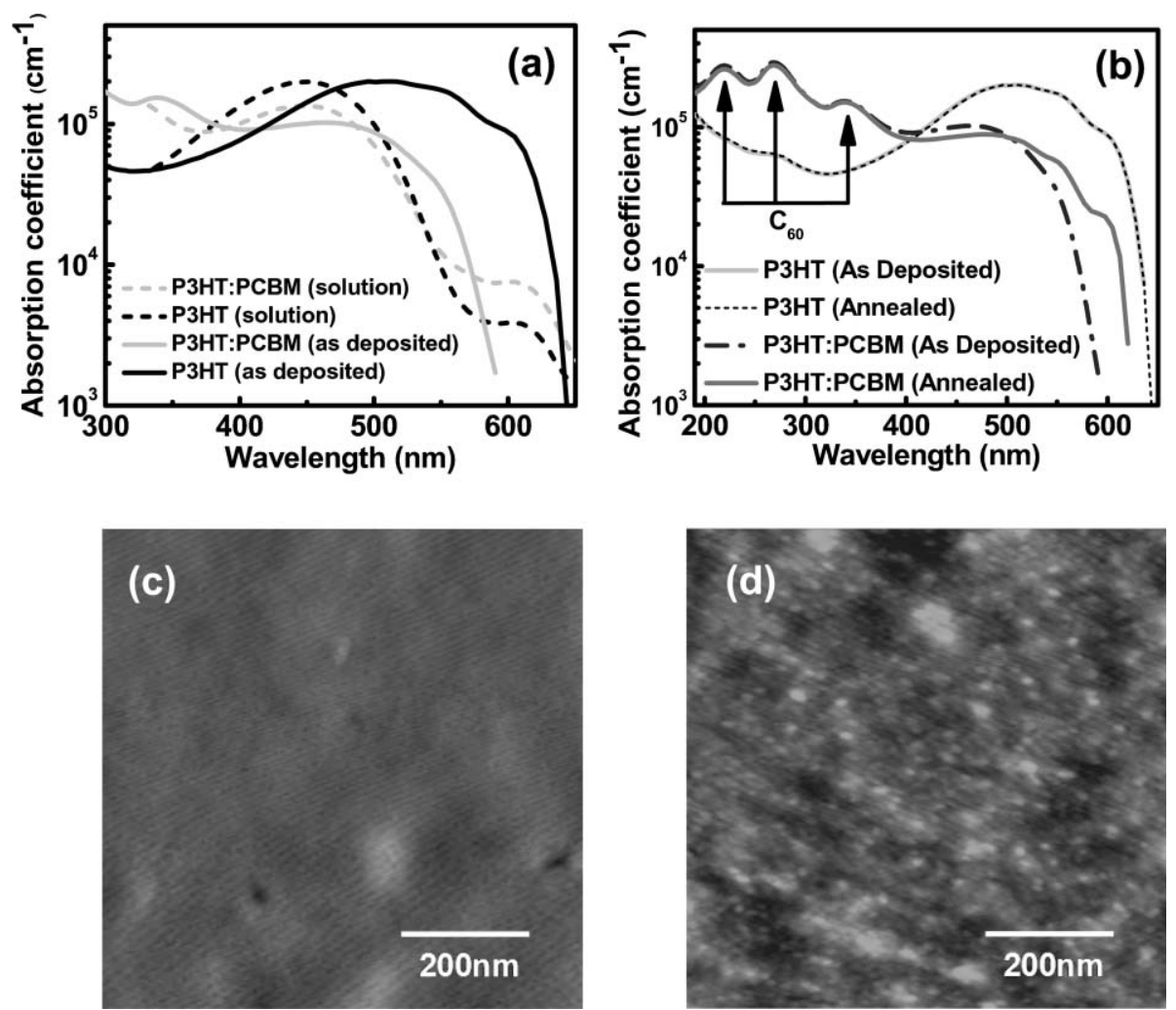

Fig. 3 (a) Comparison of absorption spectra of P3HT and P3HT : PCBM in solution and as deposited thin solidified films. (b) Comparison of absorption spectra of as deposited thin films and solvent annealed thin films of P3HT and P3HT : PCBM. AFM height images of (c) as deposited and (d) solvent vapor annealed P3HT : PCBM thin films.

deposited thin films appear featureless while detailed topographical analysis of the solvent vapor annealed films revealed that clusters of 10-20 $\mathrm{nm}$ are prominent on the surface. The AFM image after solvent vapor annealing exhibiting more surface features than the as deposited films is shown in Fig. 3d. Although the topography in Fig. $3 d$ is not absolutely conclusive, the image is comparable to those reported in the literature ${ }^{1,12}$ for films showing dramatically improved efficiencies. It should be noted that no change in morphology by AFM upon solvent vapor annealing was observed in pure P3HT films which always appear smoother.

This interpretation of the optoelectronic spectra and the AFM images is consistent with a similar explanation for structural modifications of P3HT : PCBM during thermal annealing. ${ }^{7,17}$ In particular, Yang et al. ${ }^{17}$ showed by means of transmission electron microscopy (TEM) that thermal annealing leads to de-mixing of P3HT and PCBM via formation of PCBM-rich regions, allowing the reorganization of the residual P3HT phase in stacked coplanar conjugated segments. The reorganization is prevented in the as deposited P3HT : PCBM films because of the rapid solidification and intercalation of PCBM. In solvent vapor annealed P3HT : PCBM however, ordering can occur via the diffusion of solvent vapor which softens the organic nanocomposite, allowing the migration of PCBM and the subsequent reorganization of P3HT. Indeed, solvent vapor annealing may be comparable to controlled evaporation of the solvent after deposition which has also been demonstrated to improve the solar cell efficiency, presumably by allowing ordering to occur. ${ }^{7} \mathrm{We}$ suspect that the observed changes in the optical absorption edge may also be due to stacking and subsequent $\pi$-conjugation of the P3HT segments.

\subsection{Photoluminescence}

The PL and Raman spectra of P3HT and P3HT : PCBM films before and after solvent vapor annealing are compared in Fig. 4a. It can be seen that the polaron-exciton binding energy in our P3HT : PCBM samples remains at $2 U \sim 0.2 \mathrm{eV}$, irrespective of the annealing time and the presence of PCBM. However, a closer examination and careful fitting of the PL curves show that a small decrease of approximately $0.015 \mathrm{eV}$ occurs with solvent vapor annealing. In P3HT, the polaron-exciton confinement parameter $(\gamma)$ can be extracted from the energy in the absorption spectrum where $\alpha=10^{4} \mathrm{~cm}^{-1}\left(E_{04}\right)$ and the correlation energy $(U)$ using the formula: ${ }^{27,33,34}$

$\gamma=\left(1-2 U / E_{04}\right) \sin ^{-1}\left(1-2 U / E_{04}\right)\left[1-\left(1-2 U / E_{04}\right)^{2}\right]^{-1 / 2}$

Substituting the energy where $\alpha=10^{4} \mathrm{~cm}^{-1}\left(E_{04}\right)$ from measurements in Fig. $3 \mathrm{a}$ and $\mathrm{b}$ and values for $2 U$ into eqn (1), $\gamma$ is found to remain relatively constant at $\sim 2.4$. Although the exciton delocalization is expected to increase with ordering, our result for the confinement parameter remaining relatively unchanged is not surprising. This can be attributed 

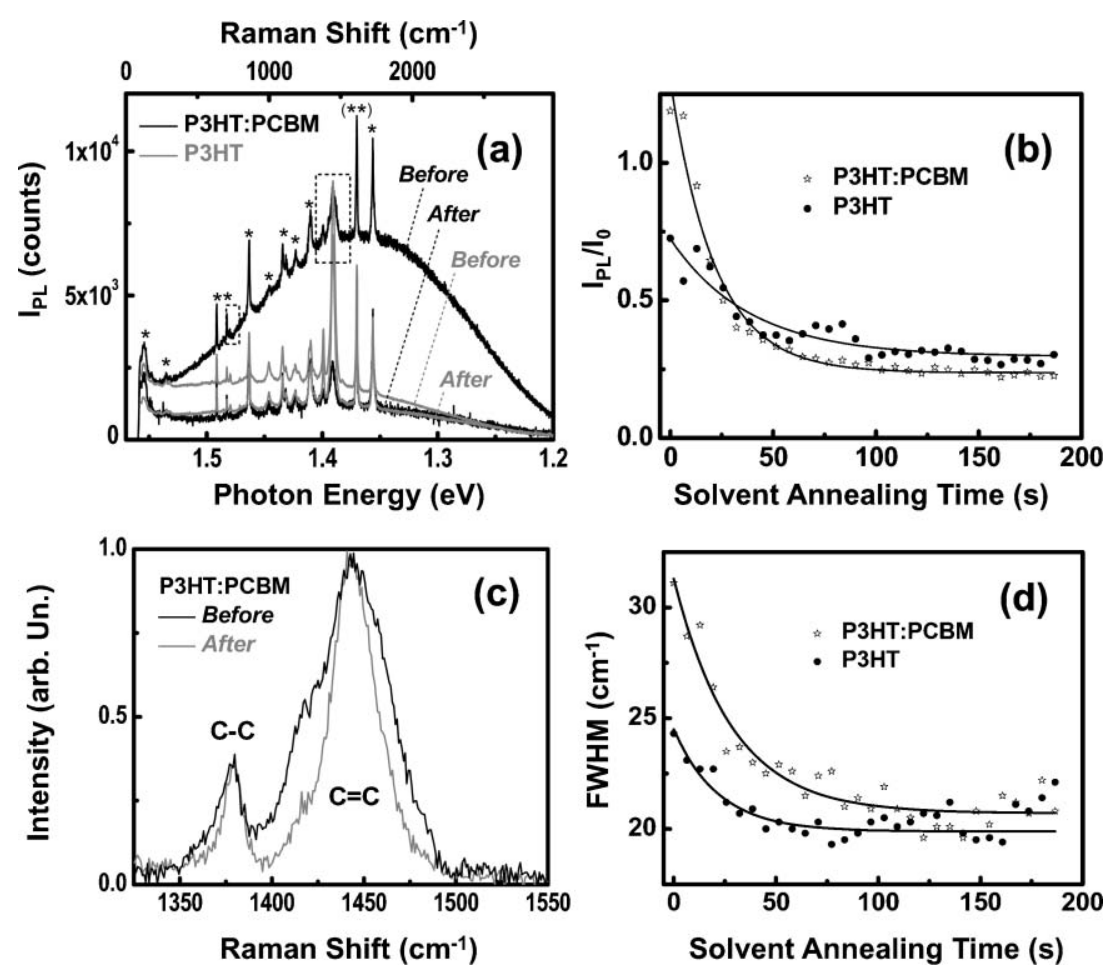

Fig. 4 (a) PL and Raman spectroscopy results of pure P3HT and P3HT : PCBM before and after solvent vapor annealing. Peaks marked with stars $\left(*{ }^{* *}\right)$ correspond to PET and ITO, respectively. The $715 \mathrm{~cm}^{-1}, 1380 \mathrm{~cm}^{-1}$ and $1440 \mathrm{~cm}^{-1}$ Raman peaks indicated by squares are assigned to the P3HT C-S-C ring deformation, $\mathrm{C}-\mathrm{C}$ skeletal stretching and $\mathrm{C}=\mathrm{C}$ ring stretching, respectively. ${ }^{36}$ (b) Summary of the PL intensity versus the solvent vapor annealing time for pure P3HT and P3HT : PCBM. (c) The $1350-1500 \mathrm{~cm}^{-1}$ Raman region of P3HT and P3HT : PCBM devices before and after solvent vapor annealing. (d) Summary of the FWHM of the $1440 \mathrm{~cm}^{-1}$ Raman $\mathrm{C}=\mathrm{C}$ peak versus the solvent vapor annealing time for pure P3HT and P3HT : PCBM.

to the fact that the PL in our solvent vapor annealed samples is very low, making it difficult to obtain precise values for $2 U$. The low PL intensity is due to the fact that the excitation energy $(1.57 \mathrm{eV})$ used here is well below the band gap of the P3HT so that the absorption is mediated by low density midgap states. Analysis using higher energy excitation (e.g. $2.41 \mathrm{eV}$ argon laser) closer to the band gap of P3HT would likely yield an increase in $\gamma$. The decrease in the values of $2 U$ and increase in $\gamma$ are generally correlated to increased delocalization with ordering similar to what we observe with solvent vapor annealing. $^{27,34,35}$ While the confinement parameter values obtained here are higher than those found in the literature, it has been shown that increased degrees of regioregularity of P3HT (from $50 \%$ to $80 \%$ regioregularity) leads to an increase in values of $\gamma \cdot{ }^{27}$ As the material used in this work is up to $98.5 \%$ regioregular, we believe these values to be reasonable.

The evolution of the PL peak intensity as a function of the solvent vapor annealing time is plotted in Fig. 4b. Unlike the degree of confinement which was not significantly affected by the presence of PCBM or by solvent vapor annealing, the PL intensity is strongly quenched by solvent vapor annealing in P3HT : PCBM films. Furthermore, the decrease in PL in the P3HT : PCBM films mostly occurs in the first minute of solvent vapor annealing, correlating to the improvement in the device efficiency. Although the PL intensity also decreases in P3HT films, the effect is less dramatic than in the P3HT : PCBM material.

\subsection{Raman spectroscopy}

Returning to Fig. 4a, a number of Raman peaks superimposed on the broad PL spectra can also be observed. Raman spectra recorded from bare substrates allow us to assign the peaks marked with stars $(*, * *)$ to PET and ITO, respectively. The $715 \mathrm{~cm}^{-1}, 1380 \mathrm{~cm}^{-1}$ and $1440 \mathrm{~cm}^{-1}$ Raman peaks indicated by squares are assigned to the $\mathrm{P} 3 \mathrm{HT} \mathrm{C}-\mathrm{S}-\mathrm{C}$ ring deformation, $\mathrm{C}-\mathrm{C}$ skeletal stretching and $\mathrm{C}=\mathrm{C}$ ring stretching, respectively. ${ }^{36}$ No Raman features attributable to PCBM, such as the $\mathrm{A}_{1 \mathrm{~g}} 1469 \mathrm{~cm}^{-1}$ mode of fullerenes, could be resolved. In order to extract additional information from the Raman spectra, we compare the $1350-1500 \mathrm{~cm}^{-1}$ region of $\mathrm{P} 3 \mathrm{HT}$ and $\mathrm{P} 3 \mathrm{HT}$ : PCBM devices before and after solvent vapor annealing in Fig. 4c. It can be seen from Fig. 4c that while the Raman shift of the $\mathrm{C}=\mathrm{C}$ peak is almost constant, interesting effects on the full width at half maximum (FWHM) of the peak can be observed. In pure P3HT, the FWHM decreases insignificantly (from $24 \mathrm{~cm}^{-1}$ to $20 \mathrm{~cm}^{-1}$ ) with solvent vapor annealing, as shown in Fig. 4d. This minor change in the FWHM is consistent with the negligible changes observed in the optical absorption spectra, sample morphology and the minor decrease in PL intensity. In contrast, in P3HT : PCBM films, the $\mathrm{C}=\mathrm{C}$ signal before solvent vapor annealing is much broader (about $32 \mathrm{~cm}^{-1}$ ) which narrows to $22 \mathrm{~cm}^{-1}$ after solvent vapor annealing, close to the $20 \mathrm{~cm}^{-1}$ FWHM of pure P3HT films. Once again, the relatively more dramatic decrease in the FWHM occurs within the first minute of solvent vapor 
annealing. Furthermore, since the Gaussian widths of the Raman lines in heterogeneous systems are a measure of their disorder, ${ }^{37}$ we can infer that solvent vapor annealing strongly reduces the disorder in $\mathrm{P} 3 \mathrm{HT}$ : $\mathrm{PCBM}$, consistent with stacking of conjugated P3HT segments, resulting in properties close to those of pristine $\mathrm{P} 3 \mathrm{HT}$.

\section{Discussion}

Based on the above data, it is plausible that solvent vapor annealing of P3HT : PCBM leads to substantial ordering in the polymer so that the optoelectronic, morphological, PL and Raman characteristics are comparable to those of highly ordered P3HT. This is due to the strong reduction in disorder via segregation of PCBM into PCBM-rich regions and stacking of P3HT chains. These structural changes have a beneficial effect on the solar cell performance (see Fig. 2) which can be explained by the enhanced transport of the carriers through ordering of the P3HT : PCBM matrix. ${ }^{17}$ The manifestation of the enhanced transport due to ordering of the P3HT layer can be seen as higher dark current in Fig. 2a (note that the high current region at positive voltages is primarily dominated by the dark current even under illumination). In addition to better transport, the modification of the morphology of the interface between PCBM and P3HT due to solvent vapor annealing will also play an important role in charge dissociation, as demonstrated by Bässler and co-workers ${ }^{38}$ and Koehler and co-workers for thermal annealing. ${ }^{39}$

The improvement in the solar cell device efficiency may also arise from an additional mechanism occurring during solvent vapor annealing. Based on our measurements, we have developed the energy diagram shown in Fig. 5 for the photovoltaic devices. In the as deposited P3HT : PCBM films (dotted line in the P3HT energy levels in Fig. 5), the presence of PCBM prevents the stacking of P3HT so that the optical gap and excitonic levels are similar to those of P3HT in solution. Specifically, assuming a mid-gap energy of $4.3 \mathrm{eV}$ for P3HT, the lowest unoccupied molecular orbital (LUMO)
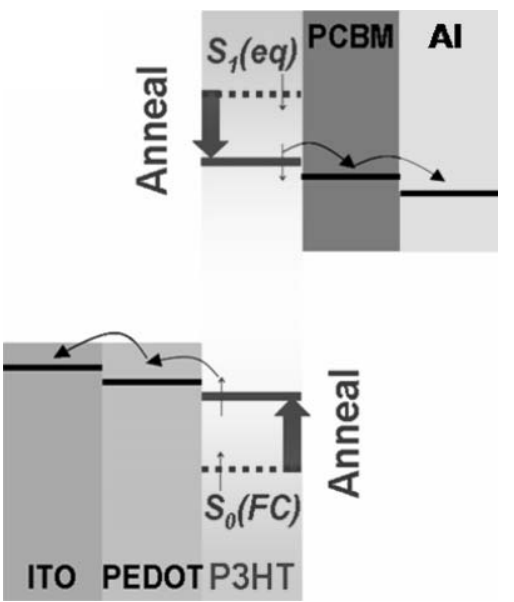

Fig. 5 Energy level diagram based on our measurements before and after solvent vapor annealing. The P3HT LUMO level shifts downward as indicated by the vertical arrows, increasing the possibility of electron transfer into PCBM and hole transfer into ITO, as indicated by the curved arrows. equilibrium level for unannealed P3HT is at $E_{1}\left[\mathrm{~S}_{1}\right.$, (eq)] $\sim 3.3 \mathrm{eV}$ compared to the acceptor level of PCBM at $\sim 3.75 \mathrm{eV}^{21,40}$ The decrease in the optical gap (from absorption spectroscopy) indicates that the LUMO of P3HT after solvent vapor annealing is closer to the acceptor state of the PCBM, as shown in Fig. 5. Thus, there will be a shift in the probability of the radiative recombination of the electron-hole pair (which creates the PL observed in Fig. 4a) to the charge transfer from the LUMO of the P3HT to the LUMO of the PCBM making the transition indicated by the curved arrows in Fig. 5 more likely. It should be noted that the shift in the LUMO of P3HT in relation to that of PCBM represented in Fig. 5 is sufficiently large to enhance the transitions indicated by the curved arrows but is sufficiently small to have any impact on the exciton dissociation. Thus, the improvement in the device efficiency with solvent vapor annealing can be described broadly by the schematic in Fig. 5. While the changes in the correlation energy $(U)$ and polaron-exciton confinement parameter $(\gamma)$ after solvent vapor annealing are not substantial, they do suggest improvement in exciton delocalization. Furthermore, additional improvement in solar cell efficiency would be provided by increased absorption between $570 \mathrm{~nm}$ and $615 \mathrm{~nm}$.

\section{Conclusions}

In conclusion, we have demonstrated that solvent vapor annealing of P3HT : PCBM at room temperature leads to a marked increase in the ordering of $\mathrm{P} 3 \mathrm{HT}$, presumably due to de-mixing of PCBM, as also observed by TEM. ${ }^{13}$ The more ordered structure provides better transport for holes through the more crystalline P3HT and electrons via hopping through the PCBM, comparable to what occurs during thermal annealing. The dissociation of excitons and ordering of the P3HT have been observed using in-situ photoluminescence and Raman spectroscopies. We discovered that the morphological changes which correlate to the improvement in the efficiencies of our solar cells occur within minutes of solvent vapor annealing. Exposure to the solvent vapor for longer periods does not change the structure or the device performance. Therefore, solvent vapor annealing might be an alternative to thermal annealing for improving the P3HT : PCBM photovoltaic performance, especially for temperature sensitive substrates.

\section{Acknowledgements}

We would like thank Prof. Adrian Mann for allowing use of the Raman instrument. We also acknowledge financial support from National Science Foundation CAREER Award (ECS 0543867). We thank the National Science Council, Taiwan for supporting Yun-Yue Lin's visit to Rutgers University.

\section{References}

1 Organic Photovoltaics: Mechanisms, Materials, and Devices, ed. S.-S. Sun and N. S. Sariciftci, Taylor \& Francis, London, 2005.

2 Organic Photovoltaics: Concepts and Realization, ed. C. J. Brabec, V. Dyakonov, J. Parisi and N. S. Sariciftci, Springer-Verlag, Heidelberg, 2003. 
3 C. J. Brabec, N. S. Sariciftci and J. C. Hummelen, Adv. Funct. Mater., 2001, 11, 15.

4 N. Camaioni, G. Ridolfi, G. Casalbore-Miceli, G. Possamai and M. Maggini, Adv. Mater., 2002, 14, 1735.

5 D. Chirvase, Z. Chiguvare, M. Knipper, J. Parisi, V. Dyakonov and J. C. Hummelen, J. Appl. Phys., 2003, 93, 3376.

6 D. Chirvase, J. Parisi, J. C. Hummelen and V. Dyakonov, Nanotechnology, 2004, 15, 1317.

7 G. Li, V. Shrotriya, J. S. Huang, Y. Yao, T. Moriarty, K. Emery and Y. Yang, Nat. Mater., 2005, 4, 864.

8 F. Padinger, R. S. Rittberger and N. S. Sariciftci, Adv. Funct. Mater., 2003, 13, 85.

9 M. Reyes-Reyes, K. Kim and D. L. Carroll, Appl. Phys. Lett., 2005, 87.

10 I. Riedel and V. Dyakonov, Phys. Status Solidi A, 2004, 201, 1332.

11 N. S. Sariciftci, L. Smilowitz, A. J. Heeger and F. Wudl, Science, 1992, 258, 1474.

12 S. E. Shaheen, C. J. Brabec, N. S. Sariciftci, F. Padinger, T. Fromherz and J. C. Hummelen, Appl. Phys. Lett., 2001, 78, 841.

13 H. Sirringhaus, N. Tessler and R. H. Friend, Science, 1998, 280, 1741

14 G. Yu, J. Gao, J. C. Hummelen, F. Wudl and A. J. Heeger, Science, 1995, 270, 1789.

15 L. J. A. Koster, V. D. Mihailetchi and P. W. M. Blom, Appl. Phys. Lett., 2006, 88, 093511.

16 T. Erb, U. Zhokhavets, G. Gobsch, S. Raleva, B. Stühn, P. Schilinsky, C. Waldauf and C. J. Brabec, Adv. Funct. Mater., 2005, 15, 1193

17 X. N. Yang, J. Loos, S. C. Veenstra, W. J. H. Verhees, M. M. Wienk, J. M. Kroon, M. A. J. Michels and R. A. J. Janssen, Nano Lett., 2005, 5, 579 .

18 K. C. Dickey, J. E. Anthony and Y. Loo, Adv. Mater., 2006, 18, 1721.

19 M. Berggren, G. Gustafsson, O. Inganas, M. R. Andersson, O. Wennerström and T. Hjertberg, Appl. Phys. Lett., 1994, 65, 1489.

20 Y. Zhao, Z. Xie, Y. Qu, Y. Geng and L. Wang, Appl. Phys. Lett., 2007, 90, 043504
21 D. Chirvase, Z. Chiguvare, M. Knipper, J. Parisi, V. Dyakonov and J. C. Hummelen, Synth. Met., 2003, 138, 299.

22 R. A. Street, Phys. Rev. B, 1978, 18, 1880.

23 R. A. Street, Phys. Rev. B, 1980, 21, 5775.

24 R. A. Street, Phys. Rev. B, 1981, 23, 861.

25 B. C. Hess, G. S. Kanner and Z. Vardeny, Phys. Rev. B, 1993, 47, 1407.

26 Y. H. Kim, D. Spiegel, S. Hotta and A. J. Heeger, Phys. Rev. B, 1988, 38, 5490.

27 B. Xu and S. Holdcroft, Macromolecules, 1993, 26, 4457.

28 A. Du Pasquier, S. Miller and M. Chhowalla, Sol. Energy Mater. Sol. Cells, 2006, 90, 1828.

29 V. Shrotriya, J. Ouyang, R. J. Tseng, G. Li and Y. Yang, Chem. Phys. Lett., 2005, 411, 138.

30 U. Zhokhavets, T. Erb, G. Gobsch, M. Al-Ibrahim and O. Ambacher, Chem. Phys. Lett., 2006, 418, 347.

31 P. J. Brown, D. S. Thomas, A. Kohler, J. S. Wilson, J.-S. Kim, C. M. Ramsdale, H. Sirringhaus and R. H. Friend, Phys. Rev. B, 2003, 67, 064203.

32 X. M. Jiang, R. Österbacka, O. Korovyanko, C. P. An, B. Horovitz, R. A. J. Janssen and Z. V. Vardeny, Adv. Funct. Mater., 2002, 12, 587.

33 A. O. Patil, A. J. Heeger and F. Wudl, Chem. Rev., 1988, 88, 183.

34 J. Ruhe, N. F. Colaneri, D. D. C. Bradley, R. H. Friend and G. Wegner, J. Phys.: Condens. Matter, 1990, 2, 5465.

35 T. W. Hagler, K. Pakbaz, K. F. Voss and A. J. Heeger, Phys. Rev. $B, 1991,44,8652$.

36 M. Baibarac, M. Lapkowski, A. Pron, S. Lefrant and I. Baltog, J. Raman Spectrosc., 1998, 29, 825.

37 M. V. Klein, in Light Scattering in Solids, ed. M. Cardona, Springer-Verlag, Berlin, 1975, vol. 8.

38 V. I. Arkhipov, P. Heremans and H. Bässler, Appl. Phys. Lett., 2003, 82, 4605.

39 M. Koehler, M. C. Santos and M. G. E. d. Luz, J. Appl Phys, 2006, 99, 053702 .

40 R. Valaski, L. M. Moreira, L. Micaroni and I. A. Hümmelgen, J. Appl. Phys., 2002, 92, 2035. 